\title{
Infection after total knee arthroplasty and its gold standard surgical treatment: Spacers used in two-stage revision arthroplasty
}

\author{
Junren Lu, Jing Han, Chi Zhang*, Yi Yang, Zhenjun Yao \\ Orthopedic Surgery, Zhongshan Hospital affiliated with Fudan University, Shanghai, China.
}

\begin{abstract}
Summary Periprosthetic joint infection (PJI) is one of the most devastating postoperative complications of total knee arthroplasty (TKA). Treatment varies depending on the type of infection, but two-stage revision arthroplasty using an antibiotic spacer is considered to be the gold standard of treatment. Several types of spacers are available at the moment, each with different benefits and indications, and these spacers may be improved in the future. The primary goals of selecting a given spacer are to locally deliver antibiotics and to preserve soft tissue. Use of an appropriate spacer subsequently decreases the difficulty of the second revision, the operating time, and ultimately the risk of postoperative complications.
\end{abstract}

Keywords: Total knee arthroplasty (TKA), infection, diagnosis, treatment, spacer

\section{Introduction}

Total knee arthroplasty (TKA), also known as total knee replacement (TKR), is one of the more common and successful orthopedic joint surgeries performed to treat degenerative diseases of the knee (1-3). The procedure is commonly performed on patients with severe osteoarthritis, rheumatoid arthritis, or pigmented villonodular synovitis with the main aim of relieving pain and joint dysfunction. Degenerative changes in the knee commonly occur in the elderly, though those changes are increasingly prevalent in younger patients due to changes in lifestyle. The procedure itself involves significant postoperative pain and involves a period of rigorous postoperative rehabilitation. Rehabilitation takes around 6 weeks and involves the use of mobility aids such as a walker, crutches, or a cane to compensate for the diminished weight-bearing capacity of the treated limb (4).

Several postoperative complications can occur after TKA, including deep vein thrombosis, fractures of the femoral shaft or periprosthetic fractures, loss of limb

Released online in J-STAGE as advance publication October 24, 2017.

*Address correspondence to:

Dr. Chi Zhang, Department of Orthopaedic Surgery, Zhongshan Hospital of Fudan University, 180 Fenglin Road, Xuhui District, Shanghai 200032, China.

E-mail: zhang.chi@zs-hospital.sh.cn motion, knee instability, and postoperative infection, and these complications all affect the overall success of TKA in different ways (5). Postsurgical prosthesisrelated infections are rare but nonetheless one of the most devastating postoperative complications (6). Numerous factors contribute to the rate of infection; despite the development of infection control, rates of infection are still as high as 3\%(6-8).

\section{Diagnosis}

At present, there are no optimal criteria for diagnosis of a periprosthetic joint infection (PJI) after TKA (9). An accepted definition of PJI is based on the fulfilment of one of two major criteria: two positive periprosthetic cultures with phenotypically identical organisms and/or the presence of a sinus tract communicating with the prosthesis. PJI is also diagnosed if three of six minor criteria are fulfilled (Table 1): an elevated level of serum C-reactive protein (CRP) and an increased erythrocyte sedimentation rate (ESR), an elevated synovial fluid white blood cell count (WBC), changes on a leukocyte esterase test strip, an increased percentage of synovial fluid polymorphonuclear neutrophils (PMN\%), or a single positive result from histological analysis of periprosthetic tissue (9).

PJI after TKA primarily presents as symptoms and signs of infection $(6-7,10-11)$. These include pain in the affected knee joint, swelling, erythema, effusion, signs of inflammation, and persistent drainage after TKA. 
Table 1. Threshold values for the minor criteria used to diagnose acute and chronic PJI, as proposed by the International Consensus Group on Periprosthetic Joint Infection (10).

\begin{tabular}{lll}
\hline Criterion & Acute PJI $(<90$ days $)$ & Chronic PJI $(>90$ days $)$ \\
\hline C-Reactive Protein (CRP, mm/hr) & No definite threshold was determined & 30 \\
Erythrocyte Sedimentation Rate (ESR, mm/hr) & 100 & 10 \\
Synovium White Blood Cell (WBC) Count (cells/ $\mu \mathrm{L})$ & 10,000 & 3,000 \\
Synovium Fluid Polymorphonuclear Neutrophils (PMN\%) & 90 & 80 \\
Leukocyte Esterase & + or ++ & + or ++ \\
Histological Analysis of Tissue & $>5$ neutrophils per high power field in $>5$ neutrophils per high-power field & in 5 high-power fields $(\times 400)$ \\
\hline
\end{tabular}

These symptoms are accompanied by a limited range of motion (ROM) beyond what is normally expected after primary TKA. Symptoms of pain and a limited ROM differ from regular postoperative pain or lingering pain and are indications of infection.

Radiological findings may also aid in diagnosing PJI (6-7). Late-stage findings such as periosteal bone formation, scattered foci of osteolysis, and subchondral bone formation suggest the presence of a postoperative infection. The presence of a radiolucent zone around the prosthesis is not usually present in acute infections but is present in chronic infections (6-7). This particularly aids in assessing the stability of the implant and ultimately determining the method of treatment. Nuclear imaging is particularly useful in the diagnosis of PJI as its results are not affected by the metallic prosthesis. An example of an imaging technique is the triple-phase technetium-99 bone scan (TBPS), which is widely used to identify bone remodelling around the prosthesis (6). A major disadvantage of TBPS is that it is unable to distinguish between a septic knee and aseptic loosening of the implant. Fluro-deoxyglucose positron emission tomography (FDG-PET) is another nuclear modality used to diagnose PJI (6). Inflammatory cells express a high amount of glucose transporters, causing an excess of deoxyglucose that cannot be metabolized by cells and that can be identified by a scanner (12). Although expensive, FDG-PET has been reported to have a sensitivity of $91 \%$ and a specificity of $72 \%$ in the diagnosis of PJI, and results can be obtained relatively soon after the examination is finished (13).

Numerous treatment options are available for the management of PJI, but revision surgery is regarded as the best method of treatment (6-7,10-11). Revision can be performed either in one or two stages. Two-stage revision arthroplasty is regarded as the gold standard for treatment of PJI of the knee (10). Unlike one-stage revision, two-stage revision requires the patient to undergo surgical treatment in two stages and the usage of an interim spacer. Numerous studies have reported that two-stage revision is more successful at controlling infection (14-19).

At present, several versions of antibiotic spacers are available. These spacers fall into three categories: $(i)$ a spacer made of antibiotic-loaded bone cement over an endoskeleton or a standard prosthesis (ii), a spacer made from cement molded intraoperatively (iii), and prefabricated commercial spacers. These spacers are divided into articulating spacers (spacers allowing a degree of limb motion in between revision surgeries) and static spacers (spacers that immobilize the lower limb in between the two revision surgeries). In twostage knee revision, the articulating interface can be cement-on-cement, cement-on-polyethylene, or cementon-metal (7-8).

\section{Procedure}

Two-stage revision surgery is regarded as the golden standard for the treatment for PJI. This procedure can involve the use of static or articulating spacers. Numerous studies have reported that two-stage revision surgery and articulating spacers can result in a rate of infection control as high as $95 \%$ in patients with PJI (67,10-11). Hart et al. reported that using this procedure has a success rate of $87.5 \%$ even when including patients who have undergone multiple surgeries (20-21).

The first stage of the revision involves a complete debridement of all lingering sources of infection in the affected joint cavity. This includes the prosthesis used in the primary TKA, cement, and inflamed tissue within the joint cavity. An antibiotic-loaded cement is chosen over regular bone cement to provide high doses of antibiotics, usually both vancomycin and gentamycin or tobramycin, that are locally eluted within the joint cavity. This cement is used in accordance with postsurgical intravenous antibiotics that are tailored to the pathogen causing the infection. The duration of antibiotic administration is continued for at least six to eight weeks until the infection is eradicated according to routine blood test results and radiological findings. Then patients are eligible to undergo the second surgery.

Other methods of surgical treatment have their own indications and rate of infection control. Debridement and irrigation is reported to have a success rate varying from $16-80 \%$, but this particular procedure is limited to acute infections, in which the infected prosthesis is not removed (22-24). One-stage revision surgery is reported to have a success rate varying from $73-100 \%$ (24-25). However, one-stage revision surgery has a specific indication: it is performed on patients who 
are unable to withstand the physical stress of multiple surgeries and on those with an infection that is caused by a single, non-resistant, non-virulent pathogen (67,10). Arthrodesis and amputation of the affected knee is usually the last result for patients who have an uncontrolled infection, life-threatening sepsis, and/or significant bone loss that cannot be corrected surgically. Arthrodesis and amputation is not usually chosen and results in an overall decrease in the patient's quality of life $(6-7,10)$.

\section{Types of spacers}

\subsection{Static spacers}

Use of a static spacer involves the immobilization of the affected leg knee during revision surgeries. This proves to be its main disadvantage but a static spacer is particularly suitable in situations where patients present with a severe infection with obvious swelling of the soft tissues (27-28). The local delivery of antibiotics provides a stable localized concentration of the antibiotics used in the bone cement. Preservation of the joint space and infection control are required for future revision treatment.

As mentioned earlier, static spacers are used when patients present with a severe uncontrolled infection or when patients have ligamentous instability, insufficient extensor mechanisms, a compromised soft tissue layer over the joint, or severe bone loss after removal of the prosthesis implanted during primary TKA.

A study has reported that the main advantages of static spacers are that they provide better relief for patients with infected and congested tissues and that they cost significantly less than articulating spacers (28). However, other studies have reported that static spacers yield poor postoperative limb mobility compared to articulating spacers $(6,27-28)$. A study by Emerson et al. compared patients who underwent revision surgery using a static spacer to those who underwent that surgery using articulating spacers (29), and the study found no marked differences in infection control for static spacers (7.7\%, 2 out of $26 \mathrm{knees)}$ and articulating spacers (9.1\%, 2 out of 22 knees) $(P=0.8)$. However, the study did find marked difference in limb mobility outcomes after the second revision: static spacers resulted in an ROM of $93.7^{\circ}$ and articulating spacers resulted in an ROM of $107.8^{\circ}$. A systematic review by Voleti et al. compared the results of using static and articulating spacers in revision treatment and it noted similar results (30). Voleti et al. analyzed a combination of level III and level IV studies that involved a total 1,526 patients. Static spacers were used to treat 654 patients and articulating spacers were used to treat 857 . None of the reviewed studies noted any marked difference in infection control $(12 \%$ for static spacers and $8 \%$ for articulating spacers), but they did note significant differences in the postoperative ROM
( $91^{\circ}$ for static spacers and $101^{\circ}$ for articulating spacers).

\subsection{Articulating spacers}

The main advantage of articulating spacers is that they allow motion of the affected knee in between revision surgeries. This further facilitates recovery of limb function, as patients can continue to move the affected knee even when an interim spacer is used. As with static spacers, articulating spacers also maintain the joint space and provide a local effusion of antibiotics.

There are no apparent contraindications to the usage of articulating spacers. A review by Mazzucchelli et al. reported that articulating spacers may not be used in cases where patients are found to have inadequate soft tissue cover over the knee since it may lead to problems with wound healing (28). Numerous studies have also reported that spacer fractures are a common postoperative complication of using articulating spacers (31-32). Prefabricated commercial spacers may prove to be problematic in cases of severe bone loss after removal of the primary prosthesis. In such cases, static spacers should be chosen.

Articulating spacers have been found to result in a greater postoperative ROM with the same rate of infection control (28). These are several factors for this better outcome, though the main one is the preservation of the length and elasticity of extensor mechanisms. Ambulation in between revision surgeries also prevents tissue scarring around the knee, quadriceps shortening, and capsular thickening and contracture $(28,33)$. This subsequently facilitates future revision surgeries by reducing surgical exposure and the overall difficulty of revision (34-35). Several versions of articulating spacer interfaces are presently available: cement-on-cement, cement-on-polyethylene, and metal-on-polyethylene.

\subsubsection{Articulating spacers with a cement-on-cement interface}

An antibiotic-loaded acrylic cement is used to fabricate both the femoral and tibial components. Cement-oncement spacers are basically fabricated by a surgeon or prefabricated. The spacers typically consist of antibioticloaded bone cement, and vancomycin and tobramycin or gentamycin are usually added. Molds of different sizes are used to form the femoral and tibial components with the antibiotic mixture serving as the base. Antibiotics are added without exceeding $10 \%$ of the total weight of the bone cement to keep from compromising the structural integrity of the spacer (36-37).

In a study of 24 patients, Durbhakula et al. used custom molds to fabricate both the femoral and tibial components (38). Durbhakula et al. used antibiotic spacers loaded with $2.4 \mathrm{~g}$ of tobramycin and $1.0 \mathrm{~g}$ of vancomycin. Postoperative follow-up was conducted for up to 33 months. Postoperative ROM was found 
to be $104^{\circ}$. A study of similar hand-made spacers by Villanueva-Martínez et al. reported that ROM was an average of $80^{\circ}$ and that there were reinfections at 5 years (31).

An example of a commercial prefabricated spacer is the Interspace Knee temporary knee spacer (Exatech, Gainesville, FL. USA, sold as the Spacer-K (Tecres, Verona, Italy) in Europe). This spacer has been approved by the US Food and Drug Administration (FDA) for use in the treatment of PJI in two-stage revision surgeries (27). However, the antibiotic component of the Interspace Knee spacer has proven to be a major disadvantage (39). The spacer is available in three sizes with gentamycin dosages ranging from $0.8 \mathrm{~g}$ to $1.7 \mathrm{~g}$, which is significantly lower than the suggested dosage of $3.6 \mathrm{~g}$ antibiotic per $40 \mathrm{~g}$ of bone cement. Another prefabricated gentamycin/vancomycin-loaded cement spacer (Spacer-K ${ }^{\circledR}$ or Vancogenx-Space Knee ${ }^{\circledR}$, Tecres, Sommacampagna, Italy) is also available. This spacer is available in four sizes: small, medium, large, and extra-large (60-, 70-, 80- and 90-mm tibial plateau dimensions, respectively). These spacers are also preloaded with antibiotics by the manufacturer.

A variation on this type of spacer involves the addition of a prosthetic stem into both the tibial and femoral intramedullary canals. The prosthetic stem is fabricated using $3 \mathrm{~mm} \mathrm{~K}$-wires that are then coated with an antibiotic cement mixture. Only the proximal part of the stem and the prosthesis component are fixed onto the bone surface.

\subsubsection{Articulating spacers with a cement-on-polyethylene interface}

In a study of 28 patients, Evans et al. used $40 \mathrm{~g}$ of Palacos R cement (Zimmer) along with $4.8 \mathrm{~g}$ of tobramycin and $4.0 \mathrm{~g}$ of vancomycin to fabricate articulating cement spacers (40). The cement spacer was either hand-made or prepared using a disposable mold. A stemmed, posterior-stabilized, polyethylene femoral component was coated with the prepared cement mixture and implanted as an interim spacer. Evans et al. obtained a success rate of up to $75 \%$ in the knees that were operated on. Few studies have described the use of this type of spacer, and the overall feasibility of this type of spacer needs to be studied further.

A possible benefit of this type of spacer is the omission of the tibial component, thus reducing operating time and surgical difficulty. A shorter operating time increases the overall success of this method of treatment.

\subsubsection{Articulating spacers with a metal-on-polyethylene interface}

The earliest record of a procedure using these spacers is a review by Hofmann et al. in 1995 (41). A study of 26 patients with PJI performed two-revision arthroplasty using a spacer with a cement-on-polyethylene interface. An articulating spacer is fabricated by autoclaving the removed component. The re-sterilized femoral component is reinserted during the same surgery. The femoral component articulates with a new polyethylenetibial component instead of a tibial component made of regular cement. A polyethylene patellar component with pegs removed is used in $40 \%$ of the patients. The antibiotic cement used was Simplex-P cement (Howmedica, Rutherford, NJ) along with tobramycin, mixed at a ratio of $4.8 \mathrm{~g}$ of tobramycin to $40 \mathrm{~g}$ of cement. This method of cement preparation is used to fabricate the femoral, patellar, and polyethylene inserts for the tibial component.

In a study of 26 patients by Hoffman et al. (41), reimplantation was successful in all but one patient who died of non-spacer related complications. The overall follow-up for those patients was 31 months (range: 1270 months). None of the patients had poor results, and $72 \%$ of the patients had excellent results. Postoperative ROM increased an average of $30^{\circ}$ in terms of the arc of motion. No problems with wound healing, deep vein thrombosis, or pulmonary emboli were noted.

PROSTALAC $^{\circledR}$ (prosthesis with antibiotic-loaded acrylic cement, DePuy Synthes, Warsaw, IN) is a commercial prefabricated spacer with a metal-onpolyethylene interface that has been available since 1987 . The spacer consists of tibial and femoral components, both consisting of antibiotic-loaded cement, along with a bicondylar metal shell on the femoral component and a complementary polyethylene insert on the tibial component. The spacer is available in different sizes and thicknesses (42).

\section{Conclusion}

Numerous versions of arthroplasty spacers are available for clinical use. At present, there is no clear consensus on the best way to select a spacer. There are no apparent differences in infection control by static and articulating spacers, but numerous studies have found that articulating spacers yield a greater postoperative ROM. Numerous aspects must be considered to determine which type of articulating spacer will be appropriate. Patient age, severity of infection, and even cost must be included in that determination. In general, the main purpose of using a spacer in revision TKA is for effective infection control.

\section{References}

1. Van M, Nace J, Mont MA. Management of primary knee osteoarthritis and indications for total knee arthroplasty for general practitioners. J Am Osteopath Assoc. 2012; 112:709-715.

2. Pagnamo MW, Clarke HD, Jacofsky DJ, Amendola A, Repicci JA. Surgical treatment of the middle-aged patient 
with arthritic knees. Instr Course Lect, 2005; 54:251-259.

3. Ringdahl E, Pandit S. Treatment of knee osteoarthritis. Am Fam Physician. 2011; 83:1287-1292.

4. Leopold SS. Minimally invasive total knee arthroplasty for osteoarthritis. N Engl J Med. 2009; 360:1749-1758.

5. Tayton ER, Frampton C, Hooper GJ, Young SW. The impact of patient and surgical factors on the rate of infection after primary total knee arthroplasty: An analysis of 64,566 joints from the New Zealand Joint Registry. Bone Joint J. 2016; 98-B:334-340.

6. Kalore NV, Gioe TJ, Singh JA. Diagnosis and management of infected total knee arthroplasty. Open Orthop J. 2011; 5:86-91.

7. Martínez-Pastor JC, Maculé-Beneyto F, Suso-Vergara S. Acute infection in total knee arthroplasty: Diagnosis and treatment. Open Orthop J. 2013; 7:(Suppl 2:M5)197-204.

8. Cury RP, Cinagawa EH, Camarga OP, Honda EK, Klautau GB, Salles MJ. Treatment of infection after total knee arthroplasty. Acta Ortop Bras. 2015; 23:239-243.

9. Della Valle C, Parvizi J, Bauer TW, DiCesare PE, Evans RP, Segreti J, Spangehl M, Watters WC 3rd, Keith M, Turkelson CM, Wies JL, Sluka P, Hitchcock K; American Academy of Orthopaedic Surgeons. American Academy of Orthopaedic Surgeons clinical practice guideline on: The diagnosis of periprosthetic joint infections of the hip and knee. J Bone Joint Surg Am. 2011; 93:1355-1357.

10. Chun KC, Kim KM, Chun $\mathrm{CH}$. Infection following total knee arthroplasty. Knee Surg Relat Res. 2013; 25:93-99.

11. de Carvalho LH, Tempon EF, Badet R. Infection after total knee replacement: Diagnosis and treatment. Rev Bras Ortop. 2013; 24:389-396.

12. Zhuang H, Duarte PS, Pourdehnad M, Maes A, Van Acker F, Shnier D, Garino JP, Fitzgerald RH, Alavi A. The promising role of $18 \mathrm{~F}-\mathrm{FDG}$ PET in detecting infected lower limb prosthesis implants. J Nucl Med. 2001;42:44-48.

13. Reinartz P. FDG-PET in patients with painful hip and knee arthroplasty: Technical breakthrough or just more of the same. Q J Nucl Med Mol Imaging. 2009; 53:41-50.

14. Sayeed Z, Anoushiravani AA, El-Othmani MM, Chambers MC, Mihalko WM, Jiranek WA, Paprosky WG, Saleh JK. Two-stage revision total knee arthroplasty in the setting of periprosthetic knee infection. Instr Course Lect. 2017; 66:249-262.

15. Claassen L, Plaass C, Daniilidis K, Calliess T, von Lewinski G. Two-stage revision total knee arthroplasty in cases of periprosthetic joint infection: An analysis of 50 cases. Open Orthop J. 2015; 9:49-56.

16. Silvestre A, Almeida F, Renovell P, Morante E, López R. Revision of infected total knee arthroplasty: Two-stage reimplantation using an antibiotic-impregnated static spacer. Clin Orthop Surg. 2013; 5:180-187.

17. Kini SG, Gabr A, Das R, Sukeik M, Haddad FS. Twostage revision for periprosthetic hip and knee joint infections. Open Orthop J. 2016; 10:579-588.

18. Petrie J, Sassoon A, Haidukewych G. Two-stage revision for the infected total knee arthroplasty: The gold standard. Sem Arthroplasty. 2013; 24:149-151.

19. Brunnekreef J, Hannink G, Malefijt MW. Recovery of knee mobility after a static or mobile spacer in total knee infection. Acta Orthop Belg. 2013; 79:83-89.

20. Hart WJ, Jones RS. Two-stage revision of infected total knee replacements using articulating cement spacers and short-term antibiotic therapy. J Bone Joint Surg (Br). 2006; 88:1011-1015.
21. Durbhakula SM, Czajka J, Fuchs MD, Uhl RL. Antibiotic-loaded articulating cement spacer in the 2-stage exchange of infected total knee arthroplasty. J Arthroplasty. 2004; 19:768-774.

22. Bradbury T, Fehring TK, Taunton M, Hanssen A, Azzam K, Parvizi J, Odum SM. The fate of acute methicillinresistant Staphylococcus aureus periprosthetic knee infections treated by open debridement and retention of component. J Arthroplasty. 2009; 24(6 suppl):101-104.

23. Hartman MB, Fehring TK, Jordan L, Norton HJ. Periprosthetic knee sepsis: The role of irrigation and debridement. Clin Orthop Relat Res. 1991; 273:113-118.

24. Silva M, Tharani R, Schmalzried TP. Results of direct exchange or debridement of the infected total knee arthroplasty. Clin Orthop Relat Res. 2002; 404:125-131.

25. Buechel FF, Femino FP, D'Alessio J. Primary exchange revision arthroplasty for infected total knee replacement: A long-term study. Am J Orthop (Belle Mead NJ). 2004; 33:190-198.

26. Jamsen E, Stogiannidis I, Malmivaara A, Pajamki J, Puolakka T, Konttinen YT. Outcome of prosthesis exchange for infected knee arthroplasty: The effect of treatment approach. Acta Orthop. 2009; 80:67-77.

27. Jacobs C, Christian CP, Berend ME. Static and mobile antibiotic-impregnated cement spacers for the management of prosthetic joint infection. J Am Acad Orthop Surg. 2009; 17:356-368.

28. Mazzucchelli L, Rosso F, Marmotti A, Bonasia DE, Bruzzone M, Rossi R. The use of spacers (static and mobile) in infection knee arthroplasty. Curr Rev Musculoskelet Med. 2015; 8:373-382.

29. Emerson RH Jr., Muncie M, Tarbox TR, Higgins LL. Comparison of a static with a mobile spacer in total knee infection. Clin Orthop Relat Res. 2002; 404:132-138.

30. Voleti PB, Baldwin KD, Lee GC. Use of static or articulating spacers for infection following total knee arthroplasty: A systematic literature review. J Bone Joint Surg Am. 2013; 95:1594-1599.

31. Villanueva-Martínez M, Ríos-Luna A, Pereiro J, Fahandez-Saddi H, Villamor A. Hand-made articulating spacers in two-stage revision for infected total knee arthroplasty: Good outcome in 30 patients. Acta Orthop. 2008; 79:674-682.

32. Cui Q, Mihalko WM, Shields JS, Ries M, Saleh KJ. Antibiotic-impregnated cement spacers for the treatment of infection associated with total hip or knee arthroplasty. J Bone Joint Surg Am. 2007; 89:871-882.

33. Thabe H, Schill S. Two-stage reimplantation with an application spacer and combined with delivery of antibiotics in the management of prosthetic joint infection. Oper Orthop Traumatol. 2007; 19:78-100.

34. Hsu YC Cheng HC, Ng TP, Chiu KY. Antibiotic-loaded cement articulating spacer for 2-stage reimplantation in infected total knee arthroplasty: A simple and economic method. J Arthroplasty. 2007; 22:1060-1066.

35. Choi HR Malchau H, Bedair H. Are prosthetic spacers safe to use in 2-stage treatment for infected total knee arthroplasty? J Arthroplasty. 2012; 27:1474-1479. e1.

36. Masri BA, Duncan CP, Beauchamp CP. Long-term elution of antibiotics from bone-cement: An in vivo study using the prosthesis of antibiotic-loaded acrylic cement (PROSTALAC) system. J Arthoplasty. 1998; 13:331338.

37. Koo KH, Yang JW, Cho SH, Song HR, Park HB, Ha YC, Chang JD, Kim SY, Kim YH. Impregnation of 
vancomycin, gentamycin, and cefotaxime in a cement spacer for two-stage cementless reconstruction in infected total hip arthroplasty. J Arthroplasty. 2001; 16:882-892.

38. Durbhakula SM, Czajka J, Fuchs MD, Uh1 RL. Antibiotic-loaded articulating cement spacer in the 2-stage exchange of infected total knee arthroplasty. J Arthroplasty. 2004; 19:768-774.

39. Jiranek WA, Hannsenn AD, Greenwald AS. Antibioticloaded bone cement in aseptic total joint replacement: Whys, wherefores \& caveats. Presented at the 71 st Annual Meeting of the American academy of Orthopaedic Surgeons, Washington, DC, February 2327, 2005. Available online at: http://orl-inc.com/wpcontent/uploads/2016/03/ALBC-Committee-2005.pdf (accessed August 30, 2017).
40. Evans RP. Successful treatment of total hip and knee infection with articulating antibiotic components: A modified treatment method. Clin Orthop Relat Res. 2004; 427:37-46.

41. Hofmann AA, Kane KR, Tkach TK, Plaster RL, Camargo MP. Treatment of infected total knee arthroplasty using an articulating spacer. Clin Orthop Relat Res. 1995; 321:45-52.

42. Haddad FS, Masri BA, Campbell D, McGraw RW, Beauchamp CP, Duncan CP. The PROSTALAC functional spacer in two-stage revision for infected knee replacements: Prosthesis of antibiotic-loaded acrylic cement. J Bone Joint Surg Br. 2000; 82:807-812.

(Received August 8, 2017; Revised September 25, 2017; Accepted September 28, 2017) 